\title{
Hilfe, mein Patient braucht «Palliative Care»!
}

Tandjung, Ryan

DOI: https://doi.org/10.1024/1661-8157/a001878

Other titles: Help, my patient needs "palliative care"!

Posted at the Zurich Open Repository and Archive, University of Zurich ZORA URL: https://doi.org/10.5167/uzh-110277

Journal Article

Accepted Version

Originally published at:

Tandjung, Ryan (2015). Hilfe, mein Patient braucht «Palliative Care»! Praxis, 104(1):5.

DOI: https://doi.org/10.1024/1661-8157/a001878 


\section{Hilfe! Mein Patient braucht Palliative Care}

Die ambulanten Versorgungsstrukturen und Patientenströme befinden sich im Wandel und die medizinische Grundversorgung ist davon im besonderen Ausmass betroffen. Vor allem die veränderte Notfallversorgung hat spürbare Auswirkungen auf Notfallstationen. Dies äussert sich nicht nur in ansteigenden Fallzahlen, sondern in zunehmendem Masse auch durch veränderte medizinische Fragestellungen an das Personal. Während in der Vergangenheit kurative Indikationen im Zentrum standen, wird das Personal auf Notfallstationen vermehrt auch mit Patientinnen und Patienten konfrontiert, für die die potenziell kurative und maximale Therapie nicht als adäquat angesehen wird und stattdessen eine gute lindernde Therapie angebrachter erscheint. Behandlungsstrategien, die vielleicht nicht im bisherigen Verständnis einer Notfallstation anzusiedeln waren, aber bei welchen die Notfallstation bezüglich weiterführenden Massnahmen und Anordnungen (z.B. Reanimationsstatus, Intubation, intensivmedizinische Massnahmen) an einer zentralen Weichenstelle steht.

\section{Personal wünscht sich mehr Information}

Die sich veränderten medizinischen Anforderungen im Bereich palliativmedizinischer Fragestellungen auf Notfallstationen führte Klenk et al (Ref) dazu, eine Studie unter dem pflegerischen und ärztlichen Personal des universitären Notfallzentrums am Inselspital Bern durchzuführen. Die Studie bezweckte einerseits das Verständnis von Palliative Care und andererseits den Bedarf nach Unterstützung in diesem Bereich abzufragen. Die Resultate bestätigen einerseits ein sehr heterogenes Verständnis des Begriffs, aber auch dass palliativmedizinische Ansätze wenig im Widerspruch zu den eigenen Wertvorstellungen der Mitarbeitenden stehen. Viele der Befragten äusserten den Wunsch, auf frühere medizinische Angaben der Patientin/des Patienten zugreifen zu können, welche wichtige Informationen für die weitere Entscheidungsfindung liefern sollen.

\section{Ruf nach einem Konsil}

Der Umgang mit palliativmedizinischen Fragestellungen in einem Umfeld, in dem zeitliche Ressourcen knapp sind und schnelle Entscheide gefragt sind, birgt Unsicherheiten. So ist es auch wenig erstaunlich, dass viele Teilnehmende ein ständig abrufbares Palliative Care Team 
wünschen. Dieser Ruf nach einem dauerhaften Konsiliardienst für Palliativmedizin ist sehr verständlich. Der Umgang mit palliativmedizinischen Fragestellungen, schlechten Nachrichten und schwierigen Situationen sollte aber zur Kernkompetenz aller Gesundheitsfachpersonen gehören. So schwierig solche Situationen auf der Notfallstation sind und so wichtig auch die spezifischen Kenntnisse palliativmedizinischer Massnahmen sind, so lassen sich gewisse Massnahmen, z.B. das Übermitteln schlechter Nachrichten nicht delegieren. Der Fokus sollte deshalb vorab auf die entsprechende Schulung und Kompetenzförderung von allen beteiligten Mitarbeitern gelegt werden. Ein spezialisiertes Palliative Care Team spielt dabei im Wissenstransfer eine absolut zentrale Rolle. 